\title{
Implementation Mixed Wireless Network with Lower Number of Wi-Fi Routers for Optimal Coverage
}

\author{
https://doi.org/10.3991/ijoe.v17i13.24149 \\ Karrar Shakir Muttair ${ }^{1(凶)}$, Ali Z. Ghazi Zahid ${ }^{1}$, Oras A. Shareef Al-Ani², \\ Ahmed Mohammed Q. AL-Asadi ${ }^{3}$, Mahmood F. Mosleh ${ }^{2}$ \\ ${ }^{1}$ The Islamic University, Najaf, Iraq \\ ${ }^{2}$ Middle Technical University, Baghdad, Iraq \\ ${ }^{3}$ Al-Najaf Al-Ashraf International Airport, Najaf, Iraq \\ karraralnomani123@gmail.com \\ karrar.alnomanieiunajaf.edu.iq
}

\begin{abstract}
With the development of various wireless communication networks, Wi-Fi Router positioning and deployment systems have become widely popular in recent years to improve coverage in various environments. In this paper, we present an appropriate mechanism for defining the deployment of Wi-Fi Routers to improve coverage in the Oxford Languages Institute (OLI) environment. In addition, the institute's environment was simulated using the Wireless InSite (WI) Package. In this work, two types of Wi-Fi Routers are used. The first is the TP-Link, while the second is the Rocket. These two devices operate at 2.4 and $5 \mathrm{GHz}$ frequencies. There are two objectives in this work. The first aim is to determine the best location to cover the simulated scene environment in a better way. The second aim is to compare Wi-Fi Routers to find out which Wi-Fi Router is better and find out how many Wi-Fi Routers we need to cover the institute's environment. The comparison between Wi-Fi Routers was based on basic parameters to measure the performance of wireless networks, the most important of which are Coverage Rate (CR) Percentage, Signal Quality Rate (SQR), and Received Power Rate (RPR). According to the results that were shown on the Graphical User Interface (GUI) using MATLAB Software. We noticed that the CR, SQR, and RPR of the Rocket are $83.9080 \%$, $97.0082 \%$, and $-35.2337 \mathrm{dBm}$ respectively, and these results are better than the results provided by the TP-Link, as it gave the CR, SQR, and RPR are $32.1839 \%, 77.8690 \%$, and $-58.1685 \mathrm{dBm}$, respectively. Finally, we conclude that CR using the Rocket is good and we need one device to cover the institute's environment. While CR using the TP-Link is bad and we need five devices to reach the coverage provided by the Rocket because the Rocket has high transmitted power and gain capacity.
\end{abstract}

Keywords-mixed wireless network, CR percentage, SQR, RPR, optimal coverage, RSS, PLs, delay spread. 


\section{Introduction}

In recent years, emphasis has been placed on spreading wireless communications on the largest scale in various indoor environments [1]. Nevertheless, the increase in wireless communication devices, which are characterized by low cost and higher data rate services, has resulted in the provision of large quantities of operations for deploying wireless local area networks (WLANs) across a wide variety of domains [2]. Wireless Fidelity (Wi-Fi) is known as one of the best most important and widely used technologies and techniques, which has been applied in a wide variety of types for indoor environmental areas [3]. The Wi-Fi technology works in accordance with the international standards IEEE 802.11, whereby two unlicensed frequency bands 2.4 $\mathrm{GHz}$ and $5 \mathrm{GHz}$ are specified for use and linked to different Wi-Fi-based transmitters [4]. Hence, determining the best location for Access Point (AP) devices based on the calculation of length and optimizing for AP deployment because the access point is the main part of the network and its location plays a major role in improving network performance as well as optimizing coverage for various environments. In context, several algorithms and simulation scenarios have been presented by researchers in various recent researches to predict the AP device coverage area based on the local position [5]. Data transmission and receiving will be very important to measure and analyse signal strength values using various appropriate parameters to obtain good coverage areas. On the other hand, placing the fewest access points will lead to gaps in coverage, poor and insufficient performance, dead areas and failure of signals to reach the remotest areas inside or outside the buildings [6].

One of the most important aspects that many researchers have recently dealt with is determining the optimal location for AP devices. As a result, many algorithms and methods, and approaches have been introduced in order to properly recruit the AP in order to obtain the best coverage. The first method was to manually locate the AP based on a guess by the wireless network designer. However, this method has been used over a long period of time with imprecise results [7]. Other researchers presented new proposals based on mathematical models as in [8], as these models require effort, a long time, and additional complex calculations. On the other hand, several researchers have introduced algorithms for an AP deployment. As in [9], the researchers used a Multi-Purpose Genetic Algorithm (MPGA) as a way to obtain the optimal location for the AP to be deployed in order to coverage the target areas well. According to the results that emerged from the researchers, the Genetic Algorithm (GA) is imprecise in determining the appropriate location for fixation of AP, as well as it is complicated in terms of calculations and requires a long time to process. Meanwhile, other researchers in [10] used techniques supported by annealing simulation, in which models and scenarios relied on experimental propagation to obtain the length and losses of the wireless signal in indoor environments in order to enable the determination of the appropriate location to installation the access point. The main disadvantages of these presented methods are that they do not take into account the effects of materials used in the construction of various structures, the thickness of materials, and types of walls. In addition, an ideal deployment of the AP to improve coverage in an outdoor environment was presented by researchers [11]. The researchers focused mainly on the WI 
Package in the design of the interior. However, their study was limited to determining the best location for the AP for one floor only and did not take into account the impacts and losses of multiple floors [12].

Moreover, an investigation has been presented regarding the appropriate deployment of AP in indoor buildings. This investigation was carried out using Ray - Tracing methods. The focus of this work was on WI Package to design internal structures [13]. Focusing in this work on basic parameters are path losses and received power (RP) for each receiving point. Based on the results obtained by the researchers, they note that it is not possible to determine the best location for its AP when there AP more than one AP within the same building because a greater number of APs increases the losses in the paths will increase, and this will lead to a decrease in the RP for all the far receiving points deployment inside the building. In recent work, the researcher focused on localizing the access point site based on the RSS algorithm, which relies on measuring the path loss for each received point in the external network in order to determine the number of APs devices to coverage the target area well. In this algorithm, a small number of points were required to operate effectively but it may not be able to correctly identify the specific sample especially in Non-Line-of-Sight (NLOS) regions, due to the effects of collisions and the multipath reflections on the propagation of the signals transmitted from the transmitters to the receiving devices [14].

This paper focuses on the implementation and design of a mixed (Indoor and Outdoor) wireless network capable of working effectively to coverage the area and the lowest costs in order to provide communication service in the best methods with following the modern methods in implementation. This work will be carried out in three models: the first one is the simulation by using the WI Package. WI Package is an electromagnetic simulation program for the specification and design of various wireless systems for communications, networks, and others. The second model is to study the spread of signals and the most important obstacles that face the signals when sending them from the transmitting antennas to the receiving points. While the third model is a study of the best adaptation of the transmitter when changing it in more than one place within the target area to obtain the best coverage, as well as obtaining the least number of transmitters to cover the area well.

The remaining sections of the paper are organized as follows. In Section 2, the main parameters for measuring the performance of the channel for the wireless network are presented. Section 3 presents the most important parameters for measuring the performance of a wireless communication network designed in this paper. Section 4 presents the hybrid wireless network design proposal (indoor and outdoor). While Section 5 provides the best methods for presenting all the results of the paper, as well as discussing and analyzing these results accurately. Finally, conclusions and future work are presented in Section 6.

\section{Wireless channel performance measurement parameters}

Any implementation and design of an internal or external wireless network must be studied theory in detail about tracking the design stages to provide all possible solu- 
tions to accomplish this implementation in order to obtain a network capable of operating reliably and at the lowest cost. In this section, a detailed explanation will be given about the basics of signal propagation in mixed environments (indoor and outdoor) and the most important obstacles encountered by signals when sending them from the transmitting antennas to the receiving antennas. A detailed explanation of the basic parameters for measuring the performance of any wireless channel will be presented at the end of this section [15].

The characteristics of various wireless communication signals change drastically when transmitted from transmitters to receivers. Therefore, these characteristics depend largely on the distances between the transmitters and receivers. This distance between the two devices or antennas is called the channel. There are several methods and scenarios for measuring the performance of a wireless communication channel, so that this performance differs from one wireless network to another, due to the style and nature of the design of this network as well as the devices used. There are basic and necessary parameters to measure the performance of each wireless networks, and the most important of these parameters are the multiplicity of paths and the most important obstacles facing signals when moving between antennas, path losses between the two antennas, and measuring the strength of the received signals. In addition, calculate the Signal-to-Interference Ratio (SIR) that the signal encounters when it is transmitted from transmitter devices to received points in the simulated environment, and the delay time that the signal took to reach the receiving antenna [16].

\subsection{Multipath between antennas}

Multipath between antennas in wireless communication networks is the propagation phenomenon resulting from the radio signals that reach the receiving antennas in more than one path. Therefore, the path of the wireless signal when colliding with objects, these objects reflect some of these radio signals and this is called multipath as shown in Figure 1 [17]. In addition, the phenomenon of paths multiplication depends on the types and thickness of materials used in building the internal or external environments.

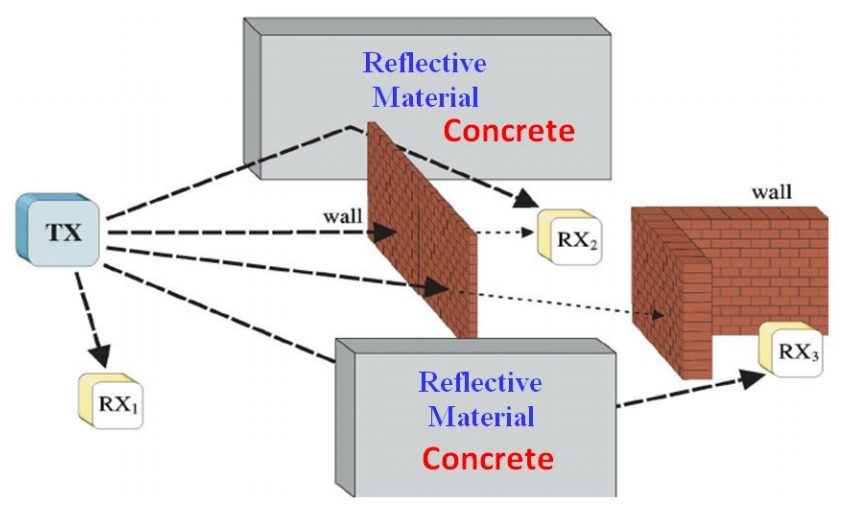

Fig. 1. The phenomenon of multiple paths. 


\subsection{Path losses (PL)}

It is an important and major component in analyzing and interpreting the correlation of various wireless communication systems. In general, the path loss is a reduction in the transmitted power from the transmitters to the receiving devices. There are many influences that affect the signal path when propagating in various environments, and the most important of these effects are materials that reflect, absorb, break and disperse the paths that travel between the antennas. In addition, after the distances between the antennas and the multipath, all of these effects cause losses, and these losses will cause the received power to decrease. To calculate the path losses through Eq. (1) [18].

$$
P L(d B)=10 \alpha\left(\log \left(\frac{d}{d_{0}}\right)\right)+20\left(\log \left(\frac{4 \pi d_{0}}{\lambda}\right)\right)
$$

Where $d_{0}$ is the reference distance in free space, $d$ is the length of distance between the transmitting antennas and the receiving points, $\lambda$ is the wavelength of the signals transmitted between the antennas, $\alpha$ is one of the exponents of $P L$.

\subsection{Received power $\left(P_{R}\right)$}

$P_{R}$ is the power decrease and dispersion of the received signals when these signals are transmitted between the transmitting and receiving antennas in free space. In general, $P_{R}$ is affected significantly by many factors and influences that cause an unexpected decrease in this strength. The most important of these influences are barriers and materials used in construction these barriers cause reflections, refractions, and collisions, and this will disperse the strength of the received power. The $P_{R}$ value for each path can be calculated by Eq. (2) [19].

$$
P_{R}(d B m)=30+10\left(\log \left(\frac{P_{t} \lambda^{2} G_{t} G_{r}}{16 \pi^{2} d^{2}}\right)\right)-L_{s}
$$

Where $L_{s}$ is an additional loss that occurs as a result of the network system's operation, $G_{t}$ is the transmission gain, $G_{r}$ is the receiving gain, and $P_{t}$ is the power of the transmitter.

\subsection{Signal-to-interference ratio (SIR)}

It is a basic and important measure in Communications and Information Technology engineering by which the desired signal level can be compared to the noise level encountered when the signal is transmitted from transmitters to receiving points. SIR is the ratio of the power received by the receiving points from the transmitter to the sum total of power from all interference sources. Therefore, it can be calculated by Eq. (3) $[19,20]$.

$$
\operatorname{SIR}(d B)=10 \log _{10}\left(P_{R}\right)-10 \log _{10}\left(I_{\text {Total }}\right)
$$

Where $I_{\text {Total }}$ is the total of the interference. 


\subsection{Delay spread $\left(\sigma_{\tau}\right)$}

It is an important measure of a wide range of effects that affect the performance of systems for different paths in the field of wireless communications. In general, it is the time difference between the arrival of the first multi-paths component and the arrival of the last path. It can be estimated and calculated by Eq. (4) [13].

$$
\sigma_{\tau}=\sqrt{\frac{\sum_{i=1}^{N_{P}}\left(\frac{L_{i}}{c}-\frac{\sum_{i=1}^{N_{P}} P_{i} t_{i}}{P_{R}}\right)^{2} P_{i}}{P_{R}}}
$$

Where $L_{i}$ is the total geometrical path length, $c$ is the light speed, $t_{i}$ is the Arrival Time (AT) for each path $i^{\text {th }}$, and $N_{P}$ is number of received point.

\section{RSS algorithm to estimate optimal coverage}

To estimate the best coverage of the simulated scene environment and how many $\mathrm{Wi}$-Fi routers the simulated environment needs to get better coverage was focused on the RSS algorithm. In this algorithm, the most important criteria and parameters to measure the performance and business scenario of the wireless network designed for the simulated scene environment were focused on improving coverage in the best way and at the lowest cost. The most important of these parameters that have been emphasized are the ratio of $\mathrm{CR}, \mathrm{SQR}$, and RPR.

\subsection{Coverage rate $(\mathrm{CR})$ percentage}

It is one of the most important basic information to determine the level of performance, functioning, and stability of any wireless communication network. In addition, this parameter focuses in its calculation on the total number of receiving points that have the best RSS to the total number of points deployed in the simulated environment scene as shown in Eq. (5) [11].

$$
C R_{(i)}=\frac{T_{H R P}}{T_{R P}} * 100 \%
$$

Where $T_{H R P}$ is the total number of received points that have the highest RSS and $T_{R P}$ is the total number of received points deployed in a simulated scene environment.

\subsection{Signal quality rate (SQR)}

It is a value that ranges from ( 0 to 100$) \%$. Whenever increase this value where the SQR of the wireless network performance is better. The SQR values depend on the theory of conditions that pass in each simulated environment, such as noise, path losses, reflections, refractions, scattering, and absorption, and the paths transmitted from the Wi-Fi Router antennas to the receiving antennas. In addition, depending on 
the performance of the systems, RSS, margin of the SNR, and wireless networking hardware you use in the simulated scene environment. To accurately estimate the values of the SQR, it can be calculated using Eq. (6) [21].

$$
S Q R_{(i)}=\frac{\sum_{j=1}^{T_{R P}}\left[2\left(P_{R(j)}+100\right)\right]}{T_{R P}} \%
$$

\subsection{Received power rate (RPR)}

It is one of the necessary parameters to determine the power performance of the received power for each receiving point deployed in various environments. In addition, by means of RPR, the power level of any transmitter can be determined. It can be calculated by Eq. (7) [21].

$$
R P R(d B m)_{(i)}=\frac{\sum_{j=1}^{T_{R P}\left(P_{R(j)}\right)}}{T_{R P}}
$$

The sequential steps followed in the RSS algorithm in order to estimate the optimal coverage in the target environment are illustrated in the flowchart as shown in Figure 2. 


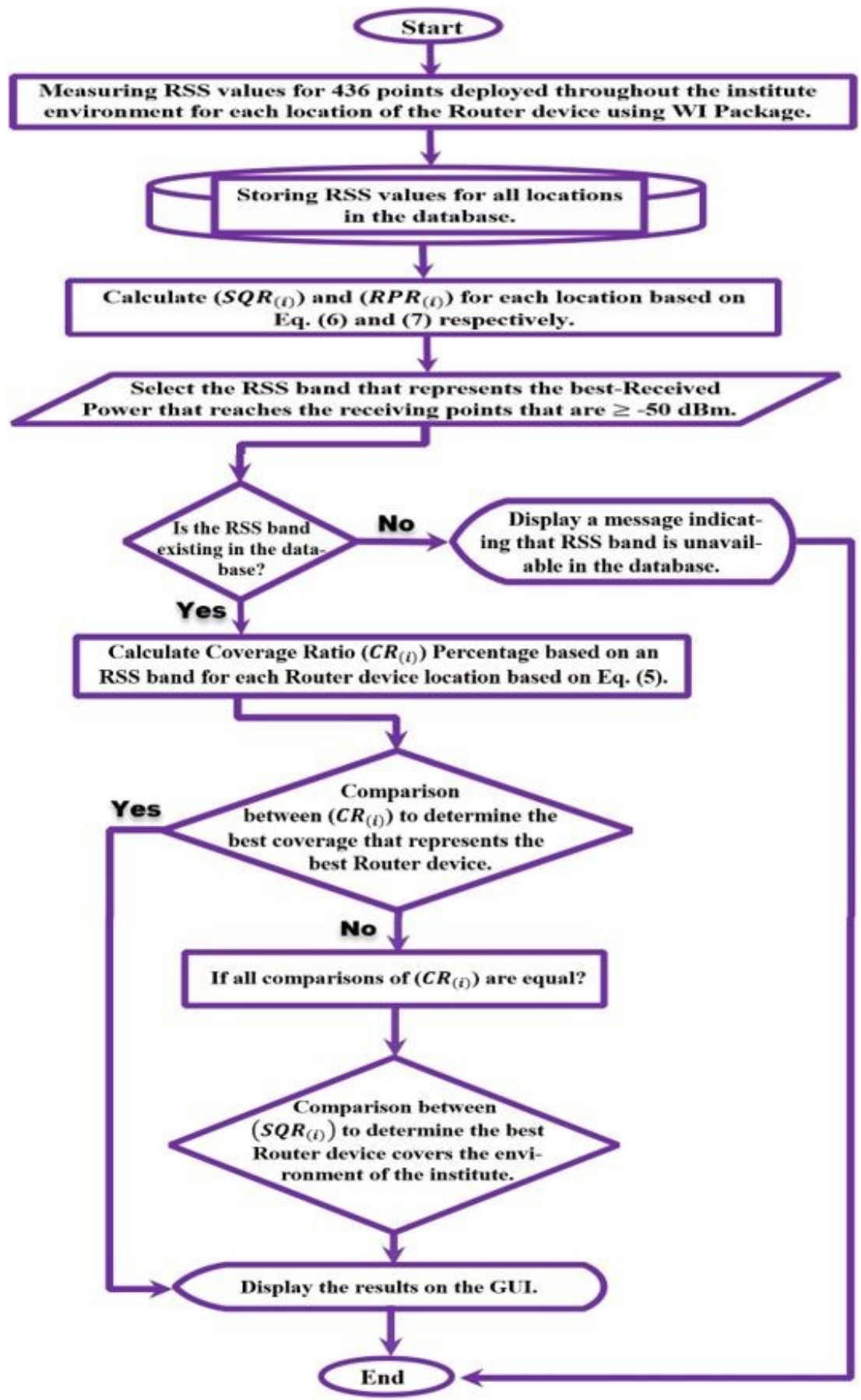

Fig. 2. The flowchart shows the sequential steps of the RSS algorithm in order to determine the best coverage in the target environment. 


\section{$4 \quad$ Proposed mixed wireless network model}

The Oxford Language Institute in Baghdad was chosen for the purposes of designing the simulation model. This institute consists of three floors (Ground Floor, First Floor, and Second Floor) as well as external gardens. The aim of this paper is to cover the institute with a good Wi-Fi network and with the least number of transmitters. Where the integration and operational performance of the network on all parts of the model operations. The building of the institute that was designed and simulated is shown in Figure 3 as a result of a simulation model using WI Package.

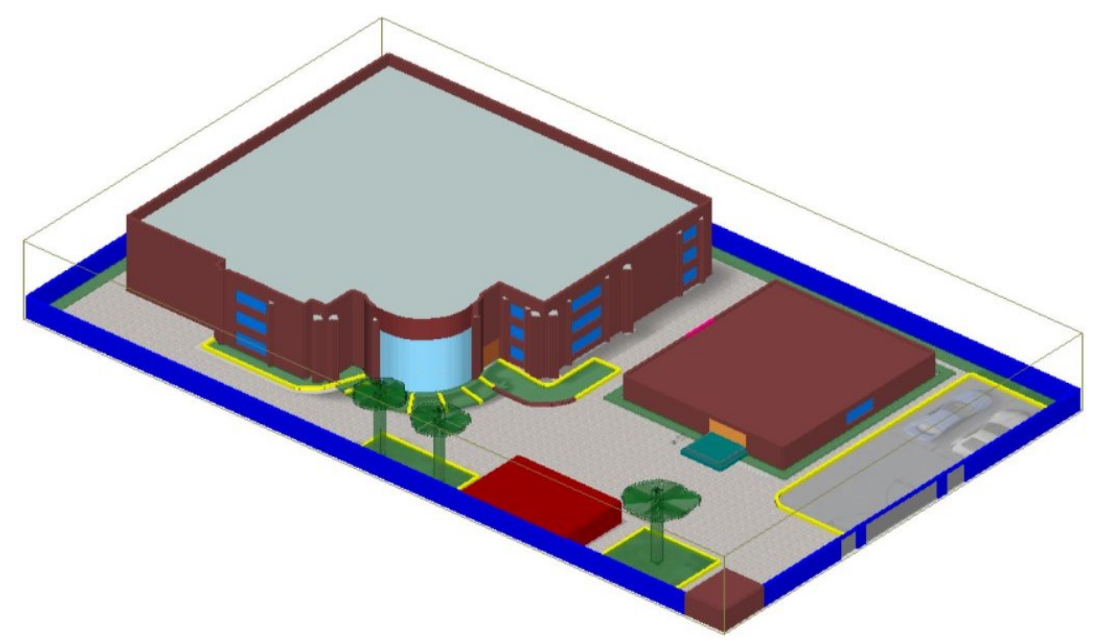

Fig. 3. Geometrical design and building model for the institute's mixed environment (indoor and outdoor).

The types and thickness of the materials used in building the institute's scene environment are listed in Table 1. The concrete material was used to build the roofs of all floors, the brick material was used to build all the walls whether internal or external of the institute, and the wood is used to design interior doors. In addition, metal is used to design the exterior doors and window frames, and glass was used for the internal frames of the windows, while dense foliage was used to decorate the earth of the institute's gardens.

Table 1. The thickness of all materials used in building the simulation scene.

\begin{tabular}{|l|c|}
\hline \multicolumn{1}{|c|}{ Materials } & Thickness (m) \\
\hline Concrete & 0.300 \\
\hline Brick & 0.280 \\
\hline Metal & 0.0625 \\
\hline Glass & 0.030 \\
\hline Dense Foliage & 0.020 \\
\hline Wood & 0.045 \\
\hline
\end{tabular}


The materials used to construct this scene using WI Package are mainly dependent on the characteristics of the electrical analog parameters. Therefore, the International Telecommunication Union (ITU) proposes that each material has conductivity and permittivity, and this conductivity and permittivity depend on the type of material as well as on the frequency used in the simulation environment $[22,23,24,25]$. The conductivity $(\sigma)$ and permittivity $(\varepsilon)$ values of the frequencies that have been used in the work environment are listed in Table 2.

Table 2. Conductivity and permittivity values of various materials used in building the simulated scene model.

\begin{tabular}{|l|c|c|c|c|}
\hline \multirow{2}{*}{ Materials } & \multicolumn{2}{c|}{$\mathbf{2 . 4} \mathbf{~ G H z}$} & \multicolumn{2}{c|}{$\mathbf{~ G H z}$} \\
\cline { 2 - 5 } & $\boldsymbol{\sigma}$ & $\boldsymbol{\varepsilon}$ & $\boldsymbol{\sigma}$ & $\boldsymbol{\varepsilon}$ \\
\hline Concrete & 0.066 & 7 & 0.119 & 7 \\
\hline Brick & 0.038 & 3.75 & 0.038 & 3.75 \\
\hline Metal & $\mathrm{e}^{8}$ & 1 & $\mathrm{e}^{8}$ & 1 \\
\hline Glass & 0.012 & 6.27 & 0.066 & 6.27 \\
\hline Dense Foliage & 0.1 & 1 & 0.1 & 1 \\
\hline Wood & 0.012 & 1.99 & 0.026 & 1.99 \\
\hline
\end{tabular}

The mixed network (internal and external) of the institute was designed by spreading the transmitters and receiving devices in the simulated integrated building environment. Two types of Wi-Fi devices were chosen, the first type is Tp-Link Router and the second type is Rocket Router as shown in Figure 4, respectively. The antennas properties of these two types of Wi-Fi are listed in Table 3. These two models were chosen in order to test which type is best for institute coverage with Wi-Fi.

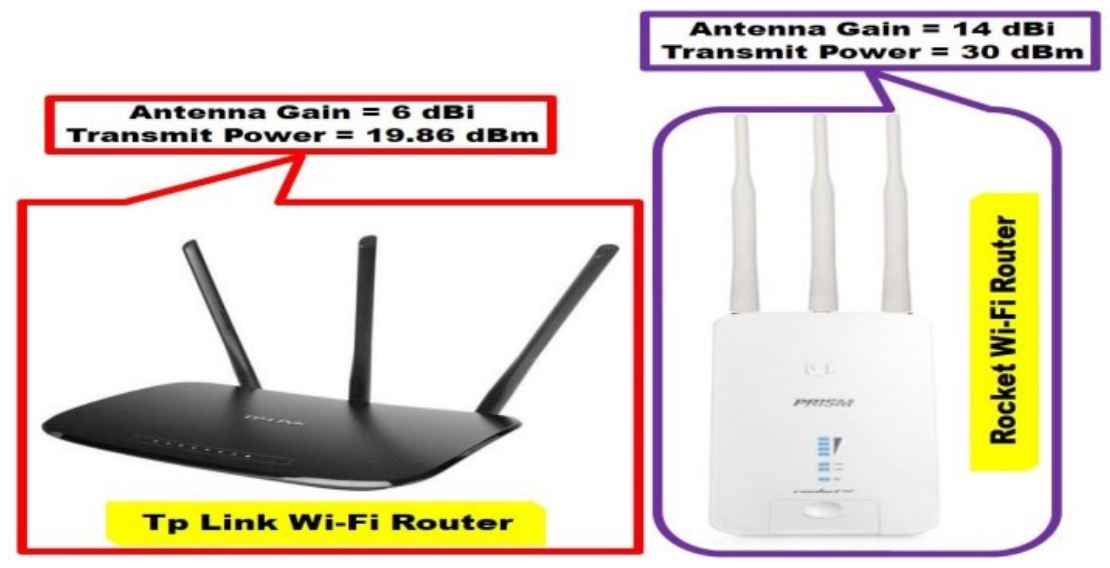

Fig. 4. The names and forms of the Wi-Fi devices that will be installed in the institute's environment. 
Paper-Implementation Mixed Wireless Network with Lower Number of Wi-Fi Routers for Optimal...

Table 3. The antennas properties of Tp-Link Wi-Fi, Rocket Wi-Fi, and Received Points.

\begin{tabular}{|l|c|c|c|}
\hline \multicolumn{1}{|c|}{ Properties } & $\begin{array}{c}\text { Tp-Link } \\
\text { Wi-Fi Router }\end{array}$ & $\begin{array}{c}\text { Rocket } \\
\text { Wi-Fi Router }\end{array}$ & Received Points \\
\cline { 2 - 4 } & $\begin{array}{c}\text { Omni } \\
\text { Directional }\end{array}$ & $\begin{array}{c}\text { Omni } \\
\text { Directional }\end{array}$ & $\begin{array}{c}\text { Omni } \\
\text { Directional }\end{array}$ \\
\hline Waveform & Sinusoid & Sinusoid & Sinusoid \\
\hline Polarization & Vertical & Vertical & Vertical \\
\hline Transmit Power & $19.86 \mathrm{dBm}$ & $30 \mathrm{dBm}$ & -- \\
\hline Receiver Threshold & $96 \mathrm{dBm}-$ & $96 \mathrm{dBm}-$ & $96 \mathrm{dBm}-$ \\
\hline Electric Field Plane Beam Width & $360^{\circ}$ & $360^{\circ}$ & $360^{\circ}$ \\
\hline Operating Frequency & 2.4 and $5 \mathrm{GHz}$ & 2.4 and $5 \mathrm{GHz}$ & 2.4 and $5 \mathrm{GHz}$ \\
\hline Bandwidth & 20 and $40 \mathrm{MHz}$ & 20 and $40 \mathrm{MHz}$ & 20 and $40 \mathrm{MHz}$ \\
\hline Antenna Gain & $6 \mathrm{dBi}$ & $14 \mathrm{dBi}$ & $2 \mathrm{dBi}$ \\
\hline
\end{tabular}

These two models of Wi-Fi devices were distributed and installed inside and outside each floor. Where one of the Wi-Fi devices was installed inside the ground floor building of the institute and the second device outdoor in the ground floor building. While the third device was installed inside the building on the first floor. In addition, the fourth device was installed inside the second-floor building. Moreover, the receiving points were deployed on the ground floor are 286 points as shown in Figure 5(a), the first floor had 75 points as shown in Figure 5(b), while the second floor had 75 points as shown in Figure 5(c). Therefore, the total received points that were deployed all over the institute are 436 receiving points. The total number of Wi-Fi devices and reception points deployed in the institute is shown in Figure 5. Wi-Fi devices and received points are installed at a height of 2 and 1.20 meters, respectively.

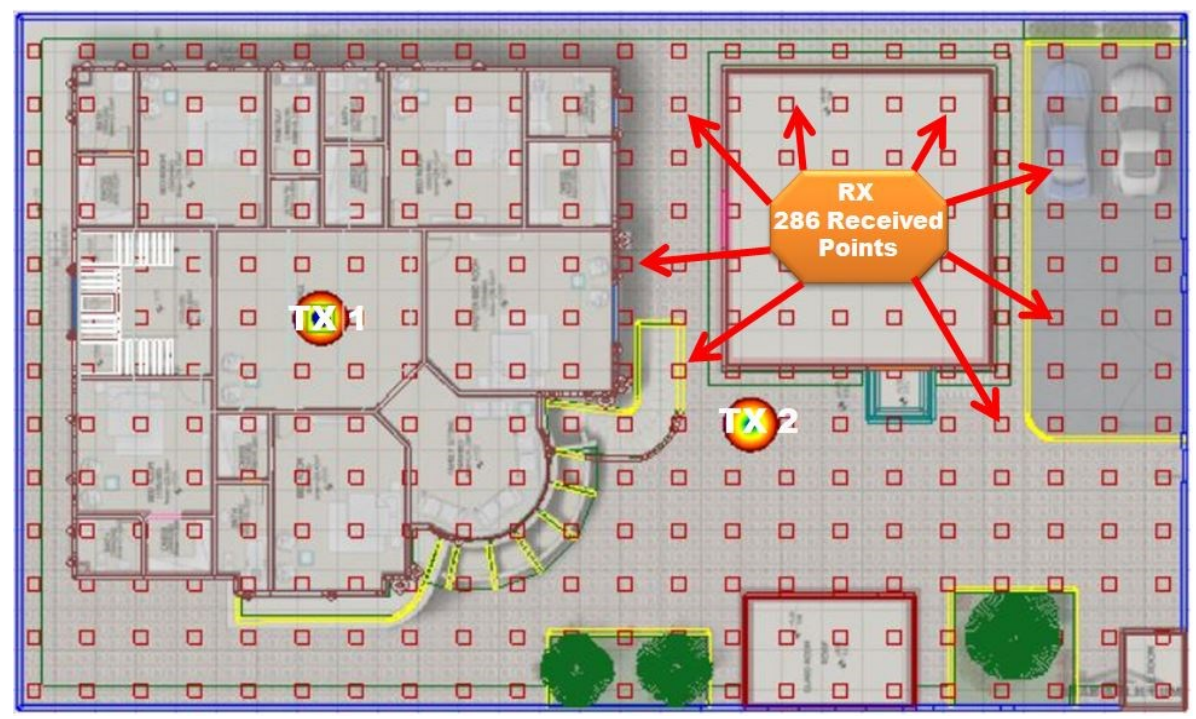

(a) Ground Floor. 


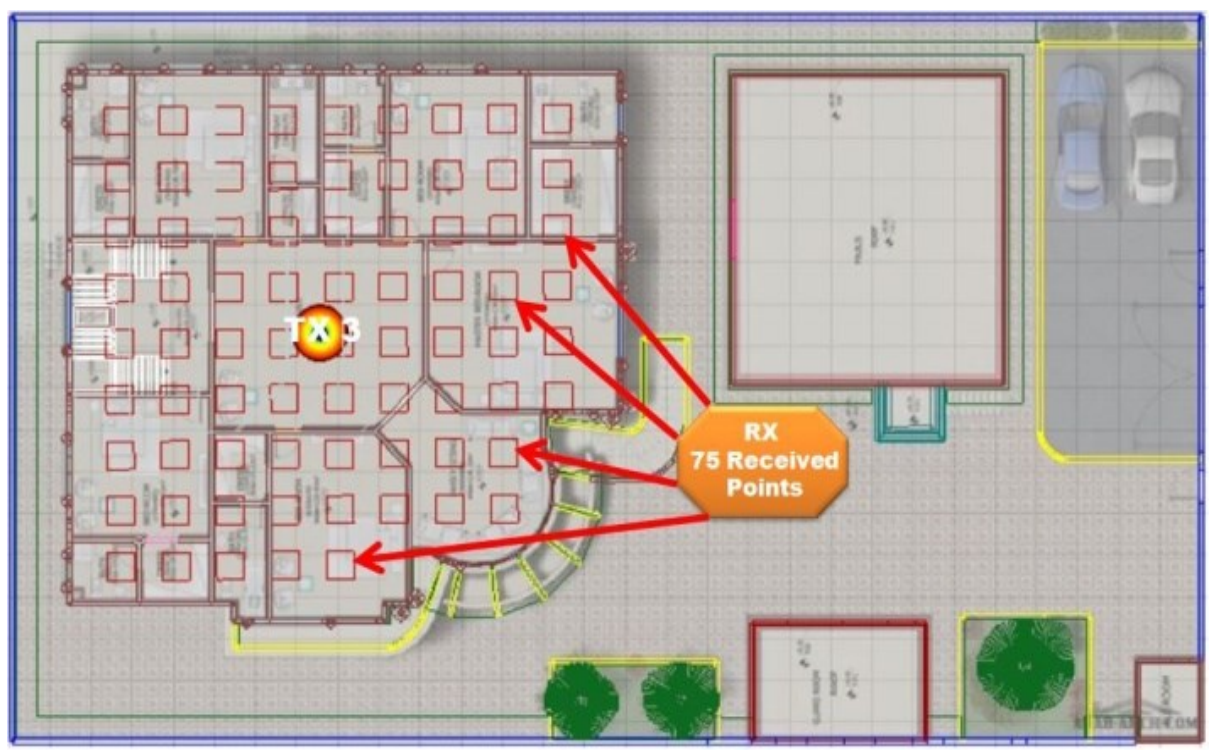

(b) First Floor.

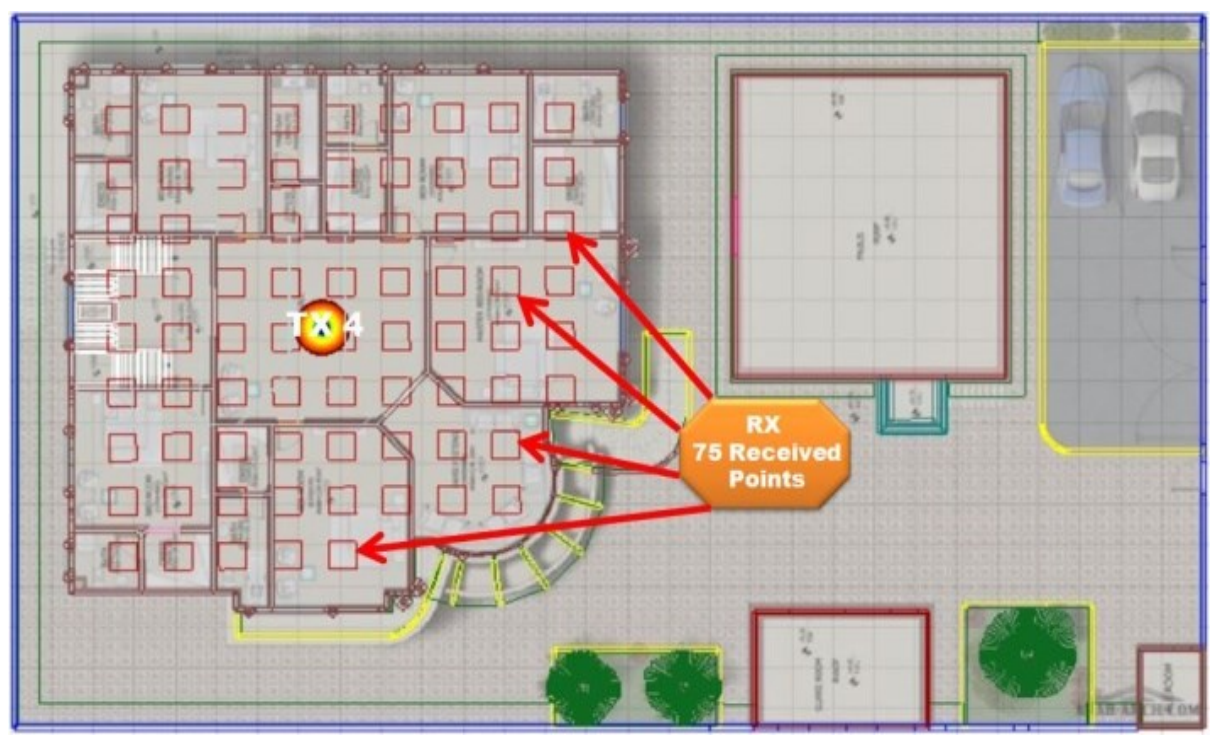

(c) Second Floor.

Fig. 5. Deploying of a Wi-Fi devices and the receiving points for each floor in the targeted institute building: (a) Ground floor, (b) $1^{\text {st }}$ Floor, (c) $2^{\text {nd }}$ Floor.

The 3D simulation design of the institute's scene environment that shows the locations of the four Wi-Fi devices spread over different floors and 436 receiving points spread over three floors is shown in Figure 6. In addition, the distance between the 
receiving point and the other is 4 meters, as these dimensions are fixed and for all points deploy whether on the ground floor, the first floor, or the second floor.

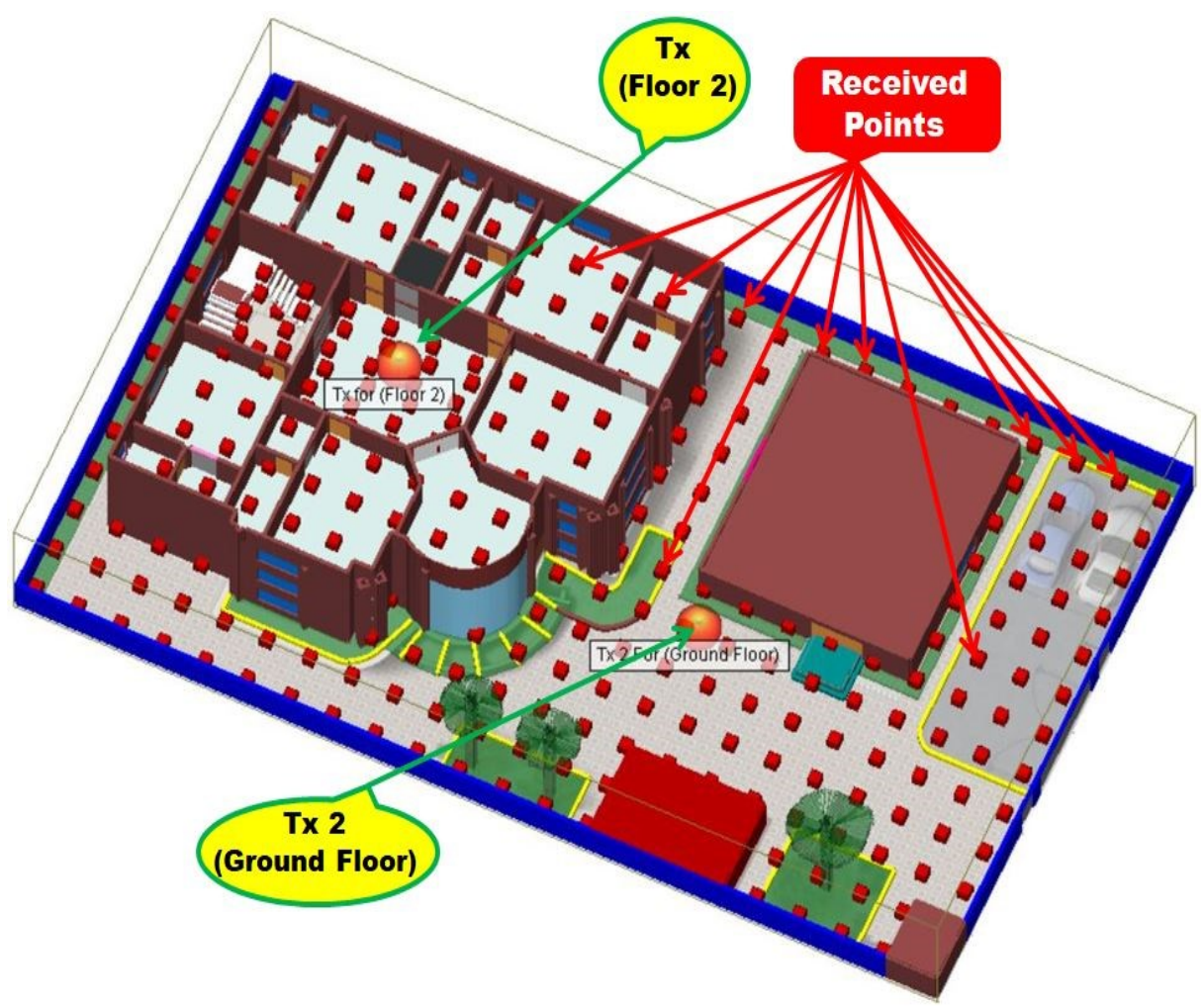

Fig. 6. The 3D simulation design that shows the locations of the Wi-Fi devices and the receiving points for the institute environment using WI Package.

Two frequencies have been simulated and worked in order to obtain the appropriate frequency to cover the institute's environment better so that frequencies are 2.4 and $5 \mathrm{GHz}$ were relied upon. Each Wi-Fi device that was installed in the simulation environment works on these two frequencies to find out which device is better to coverage the target environment appropriately and better than where Frequency, Transmit Power, and Antenna Gain. In addition, two bandwidths are 20 and $40 \mathrm{MHz}$ were used. The first bandwidth is $20 \mathrm{MHz}$ for a device that carries a frequency of 2.4 $\mathrm{GHz}$, while the second bandwidth is $40 \mathrm{MHz}$ for a device that carries a frequency of 5 $\mathrm{GHz}$.

\section{$5 \quad$ Analysis and discuss results}

The results of this section focus on measuring the performance of the signals and the most important effects and obstacles, which have encountered the signals when 
transferring from the transmitting to receiving antennas in both Line-of-Sight (LOS) and NLOS regions for two devices (Tp-Link Wi-Fi Router and Rocket Wi-Fi Router). Therefore, four main parameters are related to the performance measure of the investigated signals presenting by path propagation, delay spread, path losses, and received power.

\subsection{Delay spread}

The delay spread versus the distance for all the receiving points of the Rocket WiFi Router device for two frequencies 2.4 and $5 \mathrm{GHz}$ are shown in Figure 7. We noticed that the delay spread for two frequencies will gradually decrease as the frequency increases and distances between the Rocket Wi-Fi Router antennas and the receiving points deploying throughout the institute's scene environment. The main reason for decreasing delay spread is the presence of barriers between Rocket Wi-Fi Router devices and received points. These barriers impede the spread of signals as well as the dispersion of the signal strength transmitting from the Rocket Wi-Fi Router to the receiving antennas. There are other barriers that reflect and break the signal, such as concrete and others. In addition, we noted that the delay spread is further reduced in the NLOS regions because these regions contain a large number of inhibitors and barriers that impede the signals and absorb the strength of the signals spread in the simulated environment. Whereas in the LOS regions the decrease is much less than in the NLOS regions because the signal will pass through a smaller number in terms of barriers and walls.

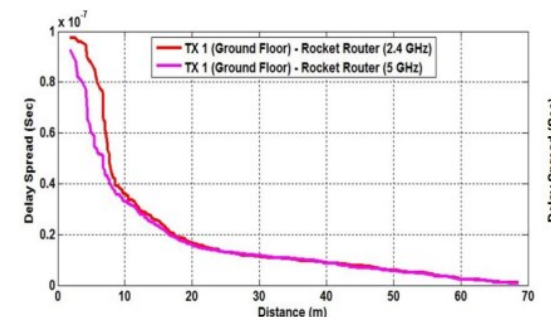

(a)

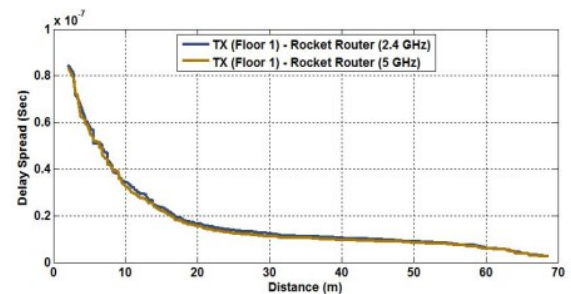

(c)

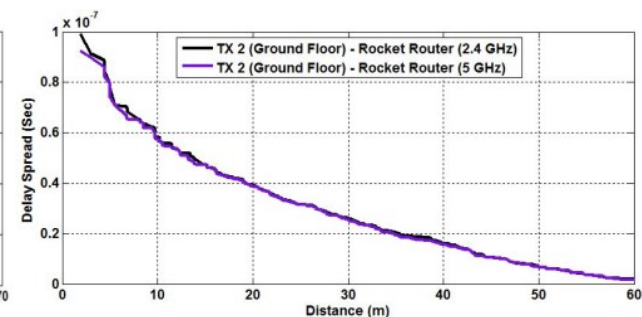

(b)

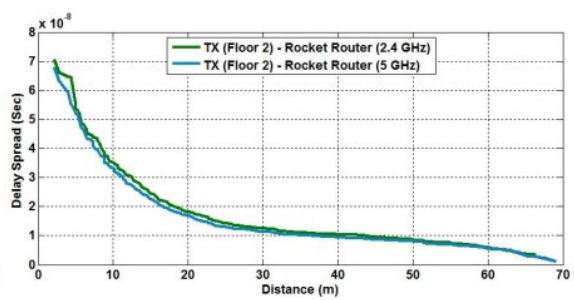

(d)

Fig. 7. The delay spread of two frequencies 2.4 and $5 \mathrm{GHz}$ versus the distance for the installed Rocket Wi-Fi device on all floors: (a) Tx 1 (Ground Floor), (b) Tx 2 (Ground Floor), (c) Tx 3 ( $1^{\text {st }}$ Floor $)$, and (d) Tx 4 ( $2^{\text {nd }}$ Floor $)$. 


\subsection{Path loss}

The relation between path loss and different frequencies versus distance for all $\mathrm{Tp}$ Link Wi-Fi Router devices installed on all floors are shown in Figure 8. We noticed that the path losses increase as the frequency increases, as the path losses at frequency 2.4 GHz have fewer losses than at frequency $5 \mathrm{GHz}$ because as the frequency increases, the wavelength of the user's frequency will be shortened. Therefore, the signal does not reach the receiving devices as quickly as possible and will suffer from not penetrating a large number of obstacles and barriers for this will increase the path's losses. In addition, we noticed that the path losses also increase with the increase in the distance between the transmitting and receiving antennas. Because as the distance between the two antennas increases, especially in NLOS regions, the signals will suffer from severe obstacles and barriers that cause absorption, refraction, reflection, or scattering of paths, all of which will increase the losses of the paths transmitted from the Rocket Wi-Fi Router device to all the receiving points deployed whether indoor or outdoor the institute's environment.

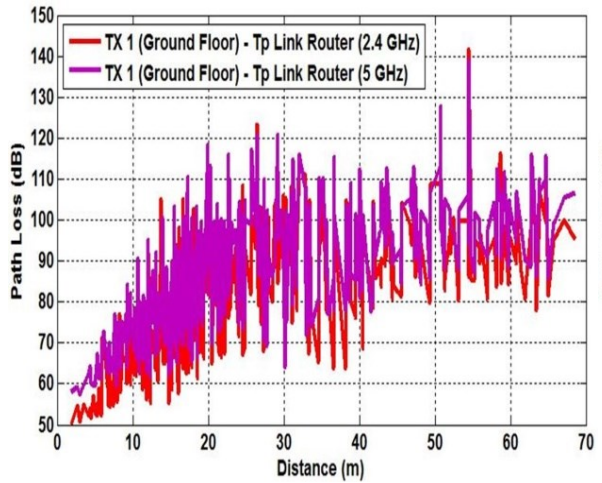

(a)

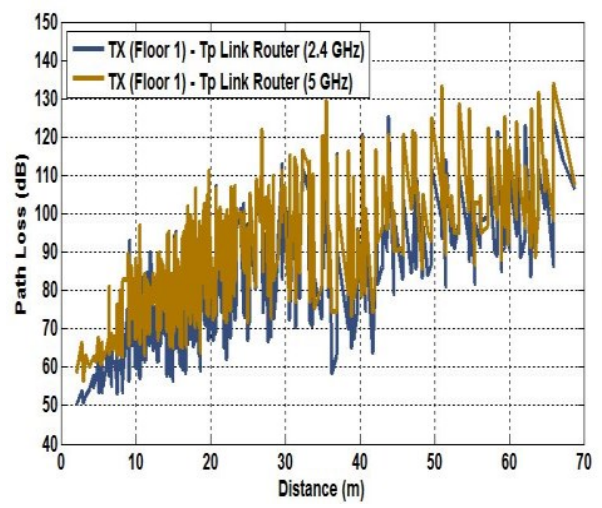

(c)

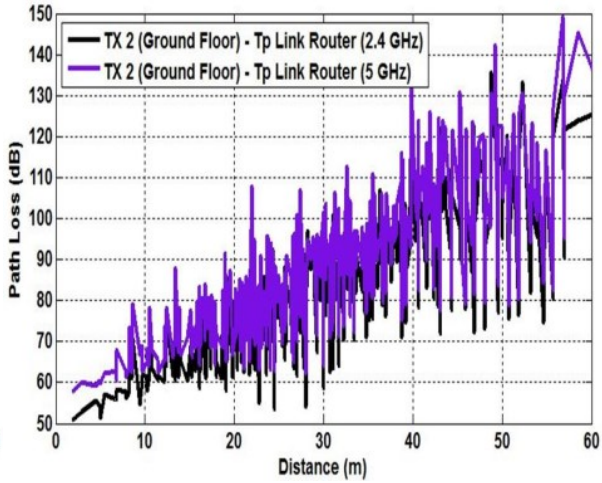

(b)

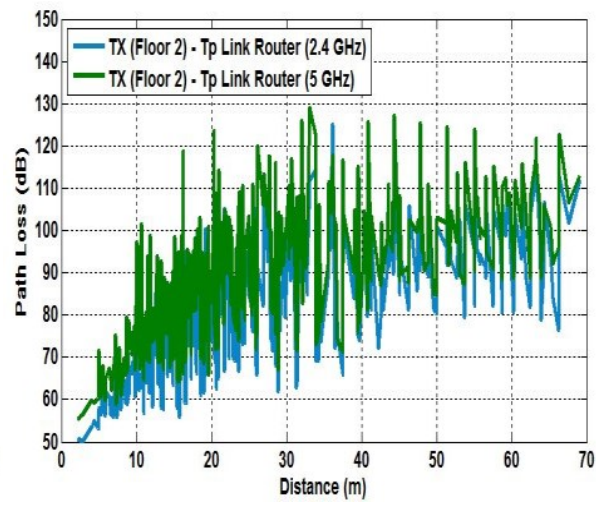

(d)

Fig. 8. The path loss for two frequencies 2.4 and $5 \mathrm{GHz}$ versus the distance for the installed TpLink Wi-Fi device on all floors: (a) Tx 1 (Ground Floor), (b) Tx 2 (Ground Floor), (c) Tx 3 ( $1^{\text {st }}$ Floor $)$, and (d) Tx 4 ( $2^{\text {nd }}$ Floor). 


\subsection{Received signal strength (RSS)}

The RSS of each received point depends on the proximity of the transmitter. There are many effects that prevent the transmitter from sending the signals to receiving points. The most important of these effects are the types of materials used in the construction that have serious effects on the signal dispersion or prevention of the signal penetration and others from obstacles. According to the results that appeared, the Rocket Wi-Fi Router for frequency $2.4 \mathrm{GHz}$ for all floors confirmed that the probability of the RSS is better compared to other locations. Because the signals transmitted from this location do not encounter large amounts of collisions and reflections. While other locations observe that the signals transmitted of the transmitter are experiencing reflections and collisions severe which will disperse the signal strength. As a result, a weak signal will arrive at the received points as shown in Figure 9. It shows the value of RSS for four locations of the Tp-Link Wi-Fi Router and Rocket Wi-Fi Router installed in the target simulation scene.

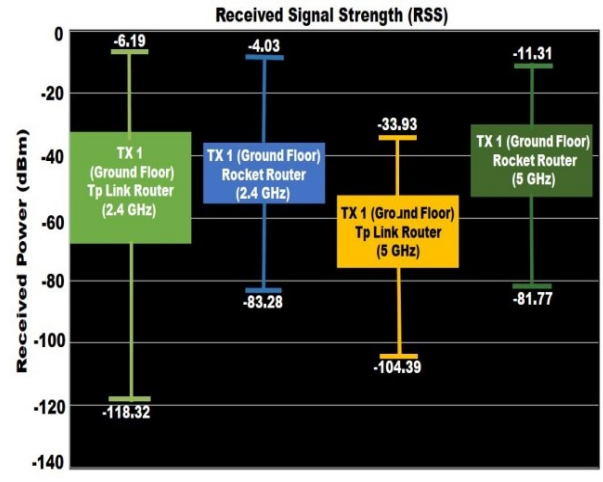

(a)

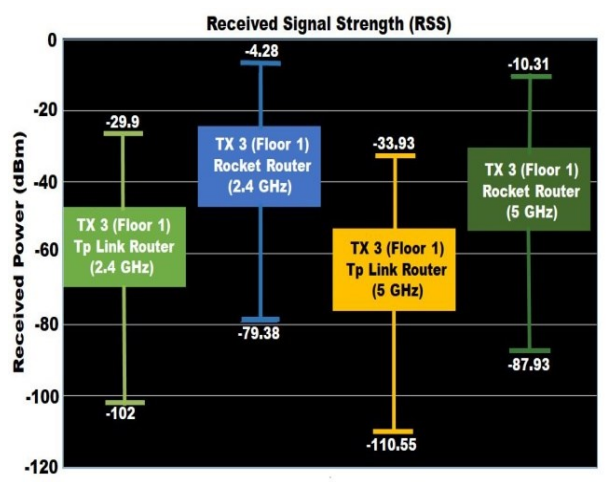

(c)

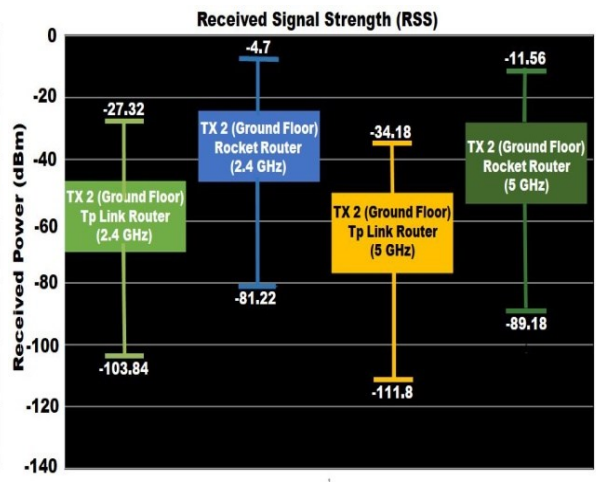

(b)

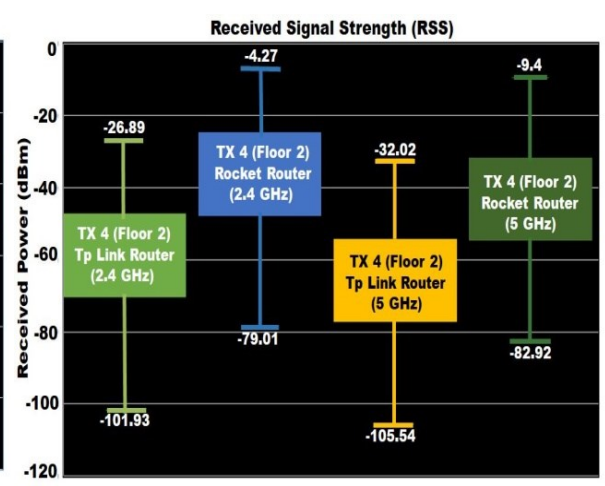

(d)

Fig. 9. The RSS values for four locations of the Tp-Link Wi-Fi Router and Rocket Wi-Fi Router for two frequencies are 2.4 and 5 GHz: (a) Tx 1, (b) Tx 2, (c) Tx 3, and (d) Tx 4. 


\subsection{Signal-to-interference ratio}

The curves of the SIR versus distance are shown in Figure 10. As shown in Figure 10 , the SIR increases with increasing distance and frequency and also increases with increasing obstacles and barriers in the simulation environment. In addition, we noticed that the SIR of the Wi-Fi Rocket Router is better than that of the Wi-Fi Tp-Link Router because Wi-Fi Rocket Router has higher power and gain, which enables it to penetrate the largest number of barriers and obstacles represented by walls. Moreover, we noticed that the SIR at the frequency of $2.4 \mathrm{GHz}$ is better than the frequency of 5 $\mathrm{GHz}$ because the higher the frequency, the shorter the wavelength of the signal carrying this frequency.

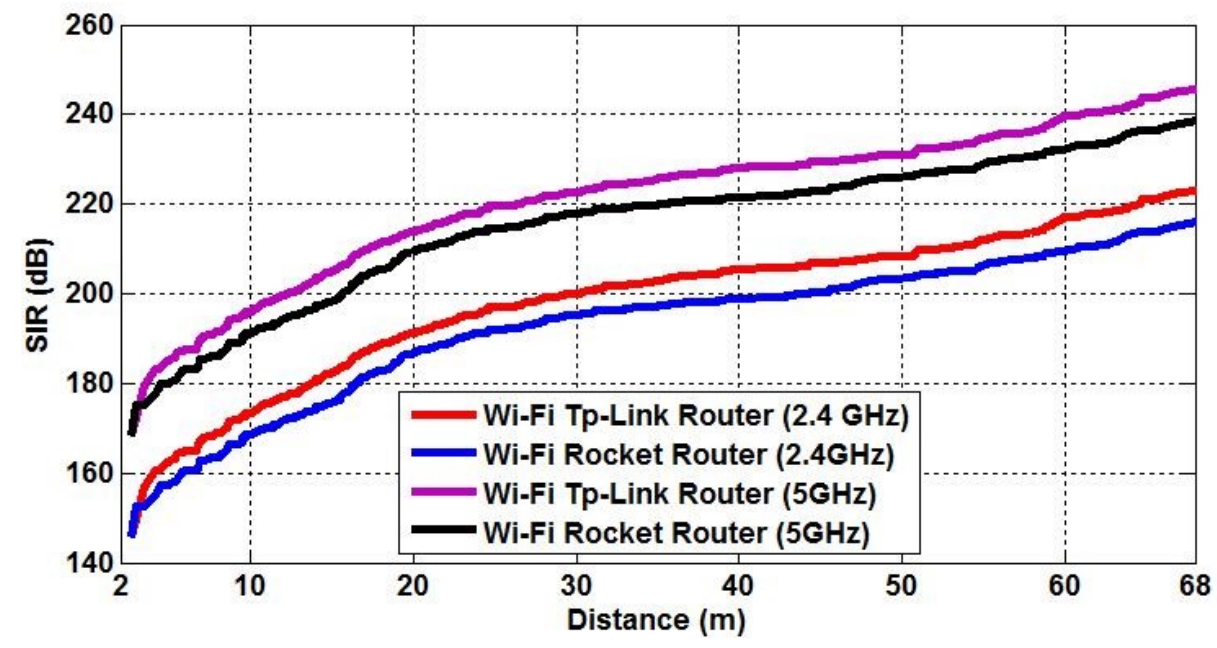

Fig. 10. The SIR curves versus distance for Wi-Fi Rocket Router and Wi-Fi Tp-Link Router for 2.4 and $5 \mathrm{GHz}$.

\subsection{Comparison of router devices performance}

The comparison between the TP-Link Wi-Fi Router and the Rocket Wi-Fi Router for a frequency of $2.4 \mathrm{GHz}$ is shown in Figure 11 , while a frequency of $5 \mathrm{GHz}$ is shown in Figure 12. The comparison relied on the most important parameters to know the performance of wireless networks, whether internal, external, or mixed. The most important of these parameters are the CR Percentage, SQR, as well as RPR for each device and frequency. The values obtained for these parameters are shown in Figure 11 and Figure 12, we note that the Rocket Wi-Fi Router is better than the TP-Link Wi-Fi Router in terms of all parameters, whether CR Percentage, SQR, and RPR for the frequency of 2.4 and $5 \mathrm{GHz}$, because the Rocket Wi-Fi Router has highest transmitted power and gain capacity from the TP-Link Wi-Fi Router. In addition, we note that the CR Percentage for the Rocket Wi-Fi Router when the input value of the RSS $\geq(-50 \mathrm{dBm})$ is $83.9080 \%$, while the TP-Link Wi-Fi Router is $32.1839 \%$ for each device deployed in an institute environment. 


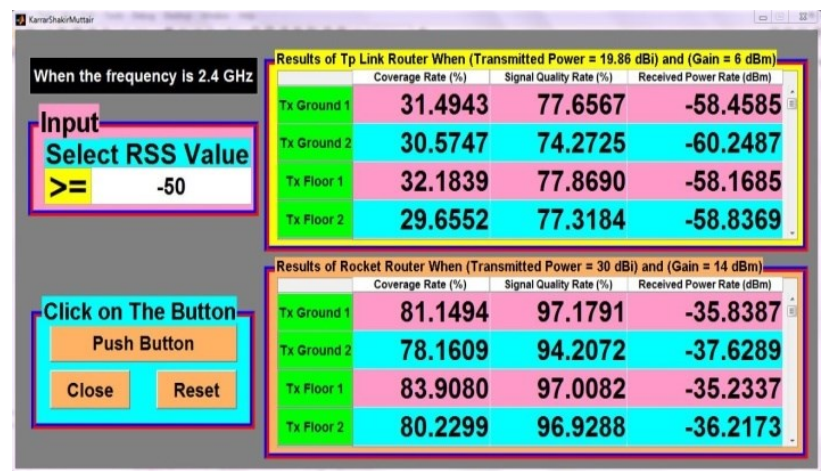

Fig. 11. Performance comparison between the TP-Link Wi-Fi Router and the Rocket Wi-Fi Router for a frequency of $2.4 \mathrm{GHz}$.

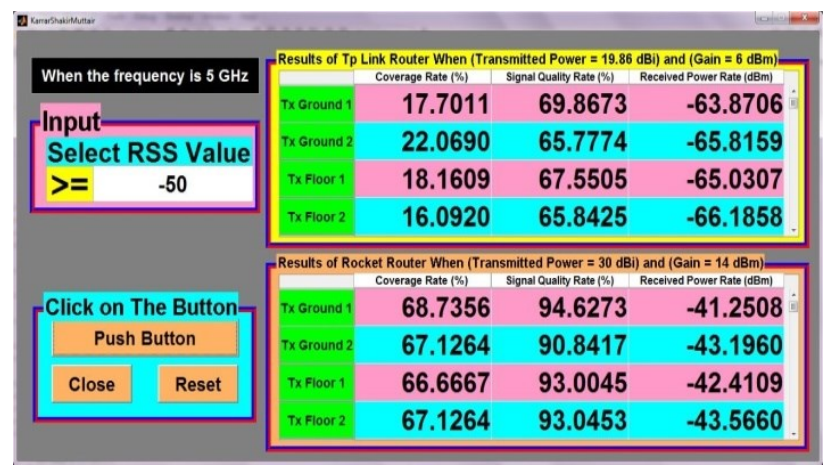

Fig. 12. Performance comparison between the TP-Link Wi-Fi Router and the Rocket Wi-Fi Router for a frequency of $5 \mathrm{GHz}$.

Moreover, if we want to cover the institute scene environment with the Rocket WiFi Router, we need one device to cover the simulated scene environment. While if we want to cover the institute's environment with the TP-Link Wi-Fi Router, you should know that we need five devices to reach the coverage of the Rocket Wi-Fi Router. Finally, we conclude that using the Rocket Wi-Fi Router to cover the institute's internal and external environment is much better than the TP-Link Wi-Fi Router in terms of coverage and lower cost. In addition, the use of five TP-Link Wi-Fi Routers will increase noise, path losses, and other influences.

\section{Conclusion}

This paper presents a new approach to identifying and deploying Wi-Fi Routers for mixed environments (indoor and outdoor). In this work, two Wi-Fi Routers were used, the first is TP-Link and the second is Rocket. These two devices operate at 2.4 and $5 \mathrm{GHz}$ frequencies. This paper relied on the most important parameters to measure the wireless network's performance which are CR percentage, SQR, RPR, Path 
Losses (PLs), Delay Spread (DS), and RSS. Two types of external and internal wave propagation characteristics are presented in this paper. The localization algorithm was implemented in three steps, the first studying the scene simulation of the target environment using WI Package and then obtaining RSS. The second step is to calculate all parameters to select the best Wi-Fi Router to coverage the simulation environment. The last step is to monitor and display the results on the GUI using MATLAB Software. Based on the results shown on the GUI, we noticed that the CR Percentage, the SQR, RPR, PLs, DS, and RSS of the Rocket Wi-Fi Router are much better than the TP-Link Wi-Fi Router. In addition, we concluded that we need one device from the Rocket Wi-Fi Router to cover the institute's environment, so that the CR percentage reaches $83.9080 \%$, while we need five devices from the TP-Link Wi-Fi Router to cover until it reaches the coverage of the Rocket Wi-Fi Router. Because the Rocket Wi-Fi Router device has a high transmitted power and gain capacity compared to the TP-Link Wi-Fi Router. Finally, in this work, we reduced the cost, noise, etc. when using the Rocket Wi-Fi Router, because as the number of routers deployed in the simulated environment increases, the cost, noise, path losses, and other influences will increase gradually.

\section{$7 \quad$ Acknowledgment}

We extend our sincere gratitude and thanks to the Islamic University in the city of Najaf / Iraq (https://iunajaf.edu.iq/ )for the continuous support for us to accomplish this academic work, so this support is considered the main gem to complete this scientific achievement.

\section{$8 \quad$ References}

[1] Yang, C. T., Chen, S. T., Den, W., Wang, Y. T., \& Kristiani, E. (2019). Implementation of an intelligent indoor environmental monitoring and management system in cloud. Future Generation Computer Systems, Vol. 96, pp. 731-749. https://doi.org/10.1016/j.future. $\underline{2018.02 .041}$

[2] So, J., \& Lee, J. (2019). Dynamic carrier-sense threshold selection for improving spatial reuse in dense Wireless LANs. Applied Sciences, Vol. 9, No. 19, p. 3951. https://doi.org/10.3390/app9193951

[3] Zhu, Q., Xiong, Q., Wang, K., Lu, W., \& Liu, T. (2020). Accurate WiFi-based indoor localization by using fuzzy classifier and mlps ensemble in complex environment. Journal of the Franklin Institute, Vol. 357, No. 3, pp. 1420-1436. https://doi.org/10.1016/j.jfranklin. 2019.10.028

[4] Vafaei, H., Kavari, G., Izadi, H. R., Dorahi, Z. Z., Dianatpour, M., Daneshparvar, A., \& Jamhiri, I. (2020). Wi-Fi (2.4 GHz) affects anti-oxidant capacity, DNA repair genes expression and, apoptosis in pregnant mouse placenta. Iranian Journal of Basic Medical Sciences, Vol. 23, No. 6, pp. 833-840. https://doi.org/10.22038/IJBMS.2020.40184.9512

[5] Puspitasari, N. F., Al Fatta, H., \& Wibowo, F. W. (2015, December). Implementation of greedy and simulated annealing algorithms for wireless access point placement. In 2015 
Paper-Implementation Mixed Wireless Network with Lower Number of Wi-Fi Routers for Optimal...

$3^{\text {rd }}$ International Conference on Artificial Intelligence, Modelling and Simulation (AIMS), pp. 165-170. IEEE. https://doi.org/10.1109/AIMS.2015.35

[6] Jia, M., Khattak, S. B. A., Guo, Q., Gu, X., \& Lin, Y. (2019). Access point optimization for reliable indoor localization systems. IEEE Transactions on Reliability, Vol. 69, No. 4, pp. 1424-1436. https://doi.org/10.1109/TR.2019.2955748

[7] Jiang, P., Zhang, Y., Fu, W., Liu, H., \& Su, X. (2015). Indoor mobile localization based on Wi-Fi fingerprint's important access point. International Journal of Distributed Sensor Networks, Vol. 11, No. 4, p. 429104. https://doi.org/10.1155/2015/429104

[8] Lee, Y., Kim, K., \& Choi, Y. (2002, November). Optimization of AP placement and channel assignment in wireless LANs. In $27^{\text {th }}$ Annual IEEE Conference on Local Computer Networks, 2002. Proceedings. LCN 2002, pp. 831-836. IEEE. https://doi.org/10.1109/ LCN.2002.1181869

[9] Maksuriwong, K., Varavithya, V., \& Chaiyaratana, N. (2003, October). Wireless LAN access point placement using a multi-objective genetic algorithm. In SMC'03 Conference Proceedings. 2003 IEEE International Conference on Systems, Man and Cybernetics. Conference Theme-System Security and Assurance (Cat. No. 03CH37483), Vol. 2, pp. 19441949. IEEE. https://doi.org/10.1109/ICSMC.2003.1244696

[10] Kondee, K., Aomumpai, S., \& Prommak, C. (2015). A novel technique for reference node placement in wireless indoor positioning systems based on fingerprint technique. ECTI Transactions on Computer and Information Technology (ECTI-CIT), Vol. 9, No. 2, pp. 131-141. https://doi.org/10.37936/ecti-cit.201592.54416

[11] Oras A. S. Al-Ani, Karrar S. Muttair, \& Mahmood F. M. (2019). Outdoor transmitter localization using multiscale algorithm. International Journal of Simulation Systems, Science \& Technology (United Kingdom), pp. 3.1-3.7. https://doi.org/10.5013/IJSSST.a.20.S1.03

[12] Iyad, H. A., Noor. A. A., Shamsul S. \& Firdaus F., (2017). Adaptive Indoor Positioning Model Based on WLAN-Fingerprinting for Dynamic and Multi-Floor Environments. Sensors, Vol. 17, No. 8, pp. 1-29. https://doi.org/10.3390/s17081789

[13] Karrar S. Muttair, Oras A.Sh. Al-Ani, \& Mahmood F. M., (2019). Outdoor MillimeterWave Propagation Simulation Model for 5G Band Frequencies. In Proc. of $2^{\text {nd }}$ International Conference on Electrical, Communication, Computer, Power and Control Engineering (IEEE), Mosul, Iraq, pp. 41-46. https://doi.org/10.1109/ICECCPCE46549.2019. $\underline{203745}$

[14] Alireza S., (2015). Evaluation and improvement of the RSSI-based localization algorithm: Received signal strength indication (RSSI), Blekinge Institute of Technology, Karlskrona Sweden. http://www.diva-portal.org/smash/record.jsf?pid=diva2\%3A844785\&dswid=-3

[15] Karrar S. Muttair, Oras A.Sh. Al-Ani, \& Mahmood F. M., (2020). Outdoor to Indoor Wireless Propagation Simulation Model for 5G Band Frequencies. In Proc. of the Fourth Postgraduate Engineering Conf., IOP Publishing, Baghdad, Iraq, Vol. 745, No. 12034, pp. 114. https://iopscience.iop.org/article/10.1088/1757-899X/745/1/012034

[16] Ahmed M. A., Tharek A. R., Marwan H. A., Nor R. Z., \& Abdallah M. S. M., (2016). Path loss model for outdoor environment at $17 \mathrm{GHz}$ mm-wave band. In Proc. of $12^{\text {th }}$ International Colloquium on Signal Processing \& Its Applications (CSPA). IEEE, Melaka, Malaysia, pp. 179-182. https://doi.org/10.1109/CSPA.2016.7515827

[17] Farahneh, H., Khalifeh, A., \& Fernando, X. (2016, May). An outdoor multi path channel model for vehicular visible light communication systems. In 2016 Photonics North (PN), pp. 1-1. IEEE. https://doi.org/10.1109/PN.2016.7537911

[18] Kim, M. D., Liang, J., Yoon, Y. K., \& Kim, J. H. (2015, July). 28GHz path loss measurements in urban environments using wideband channel sounder. In 2015 IEEE International 
Paper-Implementation Mixed Wireless Network with Lower Number of Wi-Fi Routers for Optimal...

Symposium on Antennas and Propagation \& USNC/URSI National Radio Science Meeting, pp. 1798-1799. IEEE. https://doi.org/10.1109/APS.2015.7305288

[19] S. State College Remcom Inc., \& S. Allen St. (2012). Wireless insite reference manual. IEEE Transactions on Vehicular Technology, Vol. 58, No. 1, p. 16801.

[20] Karrar Shakir Muttair, Ali Z. G. Z., Oras A. Al-Ani, Ahmed M. Q. AL-A., Mahmood F. M. (2021). A New Design of mm-Wave MIMO Antenna with High Isolation for 5G Applications. International Journal of Microwave and Optical Technology, Vol. 16, No. 4, pp. 370-379. https://ijmot.com/VOL-16-NO-4.aspx

[21] Karrar S. Muttair, Oras A.Sh. Al-Ani, \& Mahmood F. Mosleh, (2020). Performance Comparison of Multi-band Frequencies for Outdoor Communication. In Proc. of Applied Computing to Support Industry: Innovation and Technology. Communications in Computer and Information Science (Springer), Ramadi, Iraq, Vol. 1174, pp. 476-487. https://link.springer.com/chapter/10.1007/978-3-030-38752-5 37

[22] Nurminen, H., Dashti, M., \& Piché, R. (2017). A survey on wireless transmitter localization using signal strength measurements. Wireless Communications and Mobile Computing, Vol. 2017, pp. 1-12. https://doi.org/10.1155/2017/2569645

[23] Romagnoli G., Esposito G., Rizzi A., Zammori F., Bertolini M., \& Uckelmann D., (2020, May). Lab Networks in Engineering Education: A Proposed Structure for Organizing Information. International Journal of Online Engineering, Vol. 16, No. 5, pp. 41-70. https://doi.org/10.3991/ijoe.v16i05.11891

[24] Gao, G., Jia, Y., \& Xiao, K. (2018). An IOT-based Multi-Sensor Ecological Shared Farmland Management System. International Journal of Online Engineering, Vol. 14, No. 3, pp. 81-95. https://doi.org/10.3991/ijoe.v14i03.8199

[25] Jinsuo, L., Wei, Z., Dengyu, W., \& Xiaoyi, W. (2018). A New Device used in Selective Withdrawal from Reservoirs and its Effectiveness Verified in Computational Fluid Dynamics. International Journal of Online Engineering, Vol. 14, No. 3, pp. 142-151. https://doi.org/10.3991/ijoe.v14i03.8421

\section{Authors}

Karrar Shakir Muttair is a Lecturer at the Department of Computer Techniques Engineering, College of Technical Engineering, The Islamic University, Najaf, Iraq. His research interests are Computer Techniques Engineering, Computer Communications Networks, Multimedia Learning, Antennas, Indoor Wireless Networks, Outdoor Wireless Networks, Wireless Sensor Networks, and Mobile Learning. He published several types of research in the field of Communications Engineering, Networks Engineering, and Antennas. He has been awarded several awards and certificates of thanks and appreciation in the field of his work.

Ali Z. Ghazi Zahid is an Associate Professor at the Department of Computer Techniques Engineering, College of Technical Engineering, The Islamic University, Najaf, Iraq. His research interests are Computer Techniques Engineering, Computer Communications Networks, Micro-engineering, Nano-electronics, Electric, Electronic, and Computer Education field.

Oras A. Shareef Al-Ani is an Associate Professor at the Department of Computer Techniques Engineering, Engineering College, Middle Technique University, Baghdad, Iraq. Her research interests are Optoelectronic Engineering, Nanomaterial-based 
solar cell, Mobile Networks, Antennas, Indoor Wireless Networks, Outdoor Wireless Networks, and Mobile Learning.

Ahmed Mohammed Q. AL-Asadi is a work at Al-Najaf Al-Ashraf International Airport, Najaf, Iraq. His research interests are Wireless Sensor Networks, Computer Communications Networks, Mobile Networks, Antennas.

Mahmood F. Mosleh is a Professor at the Department of Computer Techniques Engineering, Engineering College, Middle Technique University, Baghdad, Iraq. His research interests are Communications Engineering, Electronic Technique, Mobile Networks, Antennas, Wireless Networks, Mobile Learning, Wireless Sensor Network, and Computer Networks and Communications.

Article submitted 2021-5-21. Resubmitted 2021-8-13. Final acceptance 2021-8-14. Final version published as submitted by the authors. 